\title{
Programación de Sistemas de Producción Híbridos, Para inventario/Bajo pedido, mediante un Proceso Analítico Jerárquico de Ordenación Grupal (GAHPO)
}

\author{
Pamela Escobar, Jaime A. Giraldo y Diana M. Cárdenas \\ Universidad Nacional de Colombia, Facultad de Ingeniería y Arquitectura, \\ Departamento de Ingeniería Industrial, Manizales-Colombia \\ (e-mail: pescobarj@unal.edu.co, jaiagiraldog@unal.edu.co,dmcardenasa@unal.edu.co)
}

Recibido Feb. 29, 2012; Aceptado Abr. 11, 2012; Versión final recibida May. 16, 2012

\section{Resumen}

Este artículo reporta la aplicación de una nueva metodología para la programación de producción en una planta industrial de la ciudad de Manizales (Colombia), en la cual se combinan los ambientes fabricación para inventario (MTS) y fabricación bajo pedido (MTO). Esto implica responder a objetivos diferentes, y en ocasiones opuestos, a partir de recursos limitados, con el consecuente impacto en el nivel de servicio. La metodología desarrollada, aplica el proceso analítico jerárquico de ordenación grupal (GAHPO), para construir una regla de secuenciación que combina tres criterios de priorización, que al ser comparada con tres reglas simples, mediante un modelo de simulación, presenta un desempeño superior en la mayoría de los indicadores seleccionados. El estudio permite concluir que es posible para empresas que operan bajo entornos de producción híbridos, lograr equilibrio entre la respuesta al mercado y la utilización de los recursos disponibles.

\section{Scheduling Hybrid Production Systems, Make to Stock/ Make to Order, using Group Analytic Hierarchy Process Ordering (GAHPO)}

\begin{abstract}
This article presents a case study of a new production scheduling methodology applied on a manufacturing facility located on Manizales, Colombia, which combines Make to Stock (MTS) and Make to Order (MTO) environments. This implies to respond, using limited resources, to different, and sometimes opposite objectives, with negative impact on the service level. The proposed methodology uses group analytic hierarchy process ordering (GAHPO) to develop a dispatching rule combining three priority criteria, that when it is compared with other three simple rules, through a discrete event simulation model, presents better performance in most of the selected measures of interest. The study allows concluding that for companies which operate under hybrid environments it is possible to reach equilibrium between market satisfaction and the utilization of available resources.
\end{abstract}

Keywords: scheduling production systems, MTS, MTO, GAHPO 


\section{INTRODUCCIÓN}

La literatura tradicional y la práctica de la gestión de la producción, han reconocido dos tipos principales de ambientes de producción: la fabricación para inventarios (make to stock - MTS) y la fabricación bajo pedido (make to order - MTO), cada uno de los cuales presenta características distintivas según la dinámica de la producción y los retos para su gestión. MTS es basado en pronósticos de demanda y se utiliza generalmente para producir productos genéricos y de alta rotación (Rafiei y Rabbani, 2009a). Por otro lado, MTO responde exclusivamente a órdenes en firme y permite mayor flexibilidad de producto, aunque con un tiempo de respuesta mayor (Chen, et al., 2009). A pesar de la clara diferenciación entre ambos, no siempre es posible evitar que aparezcan conjuntamente en los ambientes de producción reales, por lo que la literatura sobre el tema de MTS versus MTO viene desde los 60's cuando Popp (1965) presentó un modelo de un producto con inventario estocástico con lead time cero para comparar los costos simples de fabricarlo bajo MTS o bajo MTO, (Rajagopalan, 2002).

El estudio de la problemática MTS/MTO se ha mantenido relevante (Soman, 2004), pero se ha tornado más complejo a medida que la variación de producto aumenta y las condiciones del mercado son más dominantes (Kerkkanen, 2007). Durante los últimos años se ha observado una serie de cambios en las políticas de producción de las compañías y se ha visto un movimiento gradual hacia la producción híbrida MTS/MTO (Kalantari et al., 2011).

La revisión de literatura deja claro que existen diversos problemas que necesitan ser analizados en la producción en un ambiente combinado MTO/MTS. Soman (2004) divide la problemática en tres grupos de problemas: La pregunta sobre cuáles productos fabricar bajo pedido y cuáles para inventario, es la abordada con más frecuencia (Rajagopalan, (2002); Kerkkanen, (2007); Zaerpour, et al., (2008); Olhager, (2001); Soman, (2004)). Otro problema investigado es el de las políticas de producción e inventario, el cual incluye decisiones de aceptación de pedidos, determinación de fechas de entrega y niveles de inventario adecuados, asignación de capacidad y tamaños de lote (Corti, et al., (2006); Gharehgozli y Rabbani, (2006); Jiang y Geunes, (2006); Kalantari et al., (2011); Kaminsky y Kaya, (2006)). De otro lado, están las decisiones sobre la programación y control de la producción que pretenden resolver problemas de secuenciación de la producción, (Soman, et al., (2006); Chang, (2003); Federgruen y Katalan, (1999); Soman, et al., (2007); Wu, et al., (2008)). Por último, a las problemáticas identificadas por Soman (2004), se suma el "enfoque híbrido de MTS y MTO" o Make-to-Forecast (MTF) como lo llaman Meredith y Akinc, (2007). EI enfoque híbrido MTS/MTO consiste en empezar produciendo modelos de productos generales según un pronóstico (MTS) para después modificar los productos parcialmente terminados según los pedidos que vayan llegando (MTO), (Cattani, et al, (2003); Kerkkanen, (2007); Rafiei y Rabbani, (2009b)). Esta política es mencionada por algunos autores como ensamblar-bajo-pedido (assemble-to-order-ATO), terminar-bajo-pedido (finish-to-order FTO) (Olhager, 2001), producirpara-pronóstico (make-to-forecast-MTF) (Meredith y Akinc, 2007).

Meredith y Akinc, (2007) realizan una descripción de este nuevo enfoque, conceptualizándolo en diferentes industrias frente a otros enfoques de la teoría de producción. Argumentan que el reto más demandante de este enfoque es coincidir las unidades parcialmente terminadas con las órdenes de pedido entrantes, y analizar las reglas de decisión para realizar esta labor. Kalantari et al., (2011) cita a Yingdong (2001) al describir el estilo de producción MTS/MTO como un sistema en el que una porción opera en modo MTS y la porción restante en modo MTO, refiriéndose a la porción MTS como la inicial y la MTO como la final. Tsubone, et al., (2002) proponen un método para diseñar un sistema eficiente de producción combinada MTS y MTO mediante el cual la demanda insatisfecha de los mercados MTS y el tiempo de producción de los productos MTO son utilizados como medidas de desempeño de la producción. Primero analizan cómo el desempeño de la planta es afectado por la reserva de capacidad y la regla de planeación de producción, para luego presentar un método para diseñar el sistema de planeación de producción para un ambiente combinado MTS/MTO. Cattani, et al., (2003) se enfoca en la decisión de capacidad, analizando las condiciones bajo las cuales una empresa debe adoptar el enfoque dual, y determinando los niveles apropiados de "capacidad eficiente" destinada para MTS y de "capacidad flexible" para MTO. Su método consiste en restringir la capacidad eficiente para evadir exceso de inventario y separar capacidad flexible que se utilizará en modo MTO sólo cuando la demanda excede la capacidad de productos MTS. 
Contrariamente a los ambientes híbridos, en ambientes puros MTS o MTO se utilizan reglas de secuenciación simples para seleccionar el siguiente trabajo a procesar de varios trabajos que esperan del servicio de un recurso libre. La selección de la regla de secuenciación a utilizar se dificulta pues hay $n$ ! formas de secuenciar los $n$ trabajos que hay en cola para un recurso en particular y las condiciones de la planta influyen en la secuencia óptima para dicho recurso (Rajendran y Holthaus, 1999). Las reglas de secuenciación simples son normalmente destinadas a minimizar costos de inventario o tardanzas y aunque han sido aplicadas ampliamente en la práctica, su eficacia se mantiene limitada debido a su falta de visión global. Para ambientes híbridos MTS/MTO las reglas de secuenciación compuestas han demostrado ser más efectivas ya que son construidas por medio de la experiencia humana (Tay y Ho, 2008). La regla de secuenciación utilizada en la investigación de Soman et al., (2006) consiste en darle prioridad a los productos con nivel de inventario menor al inventario de seguridad definido. En cuanto a los productos MTO, debido a que su inventario de seguridad es cero, cuando una orden MTO entra al sistema, tendrá prioridad por encima de las órdenes MTS. Igualmente, en Soman et al., (2007) dan preferencia a las órdenes MTO, pero por ser un problema en el que los cambios de referencia son importantes, el método de secuenciación consiste en separar los productos a fabricar por tipo de familia, secuenciar las familias para lograr tiempos de cambio menores, y dentro de la familia, los productos son secuenciados basándose en su fecha de entrega.

Generalmente la efectividad de una regla de secuenciación simple se mide en términos de una sola respuesta, por lo que en Dabbas, et. al.(2001) proponen un modelo con el objetivo de optimizar simultáneamente múltiples respuestas de interés. Esto se logra al combinar múltiples criterios de secuenciación utilizando una combinación lineal con pesos relativos. Los pesos identifican la contribución relativa o importancia de los diferentes criterios en las respuestas objetivo. Lin, et al., (2006) utilizaron una combinación de reglas para evaluar el desempeño de una planta de fabricación de circuitos. Los resultados de la simulación sugirieron que la combinación de las reglas 'Distancia más corta', 'Producto más cerca' y 'Primero-en-entrar-primero-en-salir' logró los mejores resultados. Lin et al., (2006) citan a Hodgson y Wang, quienes estudiaron estrategias de control para un sistema híbrido Push/Pull, y sus resultados mostraron que la política híbrida logró reducir el inventario total y aumentó la fiabilidad de las entregas. En Castrillón, et. al. (2011) se proponen técnicas de inteligencia artificial para secuenciar pedidos en un ambiente puro MTO y midiendo el desempeño de la secuenciación en términos de tiempo de proceso obtenido y tiempos muertos de máquinas.

La combinación de múltiples criterios de secuenciación puede abordarse mediante un método o técnica multicriterio. El Proceso Analítico Jerárquico (AHP, por sus siglas en inglés) es un método de decisión multi-criterio que produce un vector de prioridad correspondiente a la información de preferencias del tomador de decisión, basado en comparaciones por pares de los diferentes factores de decisión. El Proceso Analítico Jerárquico fue introducido como respuesta a la necesidad de planeación y asignación de recursos militares en la década de los 70's (Zaerpour, et al., 2008). AHP ha sido ampliamente utilizado como una herramienta de toma de decisiones con criterios múltiples o como una técnica de estimación de pesos, en áreas como selección, evaluación, planeación, desarrollo y demás. El Proceso Analítico Jerárquico de Ordenación Grupal (GAHPO- Group Analyitic Hierarchy Process Ordering) es un nuevo método de ayuda para la decisión multi-criterio (MCDA), para ordenar alternativas en una decisión de grupo (Escobar, 2004). Otra particularidad de GAHPO es la incorporación de la "equidad" en la asignación de pesos a los tomadores de decisiones. Al aplicar este método, Ishizaka y Labib, (2011a) encontraron cuatro ventajas principales: reducción significativa de tiempo y esfuerzo en el proceso de decisión; facilidad para la toma de decisiones para llegar a un consenso, la mejora de la calidad de la decisión y la documentación con la justificación de la decisión tomada.

Es notable en los aportes referenciados, la estrecha relación entre las funciones de administración de capacidad y secuenciación, de tal manera que es la priorización de las diferentes órdenes de producción, la que define finalmente la manera más eficiente de asignar la capacidad disponible entre los requerimientos de producción. Las decisiones de secuenciación, por su parte, se toman en los casos documentados a partir de reglas de priorización multicriteriales. Los criterios más 
utilizados en la literatura consultada son tiempo de respuesta, fiabilidad, trúput y nivel de inventarios, los cuales guardan coincidencia con los que habitualmente son utilizados en el entorno que constituye el objeto de trabajo del presente documento. Los elementos señalados a partir de la bibliografía consultada permiten una mejor comprensión del problema que significa la gestión de la producción en ambientes combinados MTS/MTO, y servirán como bases metodológicas y conceptuales para el abordaje propuesto en el presente trabajo.

\section{PROBLEMÁTICA}

La empresa a la que se aplica el presente trabajo se dedica a la fabricación, distribución y venta de herramientas en general, principalmente para el agro, la construcción, sectores industriales y el hogar. Cuenta con una estructura de fabricación ubicada en la ciudad de Manizales (Colombia) distribuida en cinco plantas. El presente trabajo se centra en una de las cinco plantas, y su producción se distribuye aproximadamente en un 30\% para el mercado nacional y un $70 \%$ para clientes en el exterior.

El mercado nacional se atiende por medio de marcas propias las cuales son líderes del mercado, manteniendo una participación no menor del $70 \%$. A pesar de ser de menores volúmenes frente al mercado de exportación, el trúput que aportan las ventas del mercado nacional alcanza a ser hasta de un $50 \%$ del trúput total de la planta. Además, la venta del producto fabricado en esta planta es vital para el fortalecimiento del canal de distribución que permite estimular las ventas de los demás productos del catálogo. El mercado de exportación, por su parte, se compone de más de 50 países, atendidos con numerosas marcas propias y ajenas, siendo líderes en diferentes mercados. La sumatoria de estos mercados le aporta a la planta el volumen necesario para mantener una alta productividad. Las características diferentes de cada mercado obligan a que cada uno deba ser abastecido con un enfoque de producción distinto:

La fabricación de productos con destino al mercado nacional se realiza por medio de un enfoque de producción MTS debido principalmente a que:

- Las referencias exigidas por el mercado son estándares y hay poca variación en los modelos.

- Las cantidades requeridas son muy pequeñas debido a la cercanía al consumidor final.

- El tiempo de entrega requerido por los clientes es muy corto.

- Las cantidades totales de ventas se dividen en muchas referencias, lo que hace que las cantidades vendidas por referencias sean relativamente pequeñas comparadas con la capacidad diaria de la planta.

Por su parte, los productos con destino en el exterior se fabrican bajo un enfoque de producción MTO debido principalmente a:

- Se fabrican los modelos requeridos en cada país, los cuales son muy variables y con exigencias personalizadas de características específicas.

- Las cantidades y la frecuencia de los pedidos son muy variables.

- Por optimización de los costos de transportes, se despachan generalmente grandes cantidades.

La situación de tener la capacidad compartida entre productos fabricados para inventario y productos fabricados bajo pedido, permite maximizar la capacidad al mantener una producción nivelada, pero agrega una alta variabilidad en los tiempos de entrega de productos MTO y en los niveles de inventario de productos MTS, poniendo en riesgo el nivel de servicio ofrecido en ambos mercados, y, por consiguiente, la competitividad de la empresa, lo que hace justificable la búsqueda sistemática de una metodología de programación que tenga en cuenta ambos entornos en el contexto particular de la empresa seleccionada.

\section{DESARROLLO METODOLÓGICO}

Las acciones administrativas utilizadas en enfoques de producción MTS ó MTO puros son necesarias en ambientes mixtos MTS-MTO, pero la producción, en una mezcla de productos MTS y productos MTO y sus interacciones con la capacidad limitada compartida, abre posibilidades 
interesantes a problemas de programación de la producción. Por ejemplo, los productos MTS pueden ser producidos para llenar la capacidad en períodos de baja demanda de productos MTO, pero por otro lado es de gran dificultad entender completamente estas interacciones para responder preguntas como cuánto inventario se debe mantener o cómo cumplir las fechas de entrega.

En una planta típica MTS/MTO, como la seleccionada para el presente estudio, la programación de la producción se basa en un ejercicio de asignación de capacidad, en primer lugar a la fabricación de los productos requeridos para evitar la rotura de los inventarios de seguridad de los productos MTS, los cuales se priorizan según la clasificación $A B C$, y luego en los espacios de capacidad restante, se asignan los pedidos confirmados (productos MTO), que son enviados a la planta de acuerdo con una priorización que, de manera empírica, diseña el jefe de planta, con base en criterios pre-abrobados como el trúput, o la importancia del cliente.

Algunos de los problemas originados por esta metodología de programación son:

a) Sólo se considera la fecha de entrega para dar prioridad a los productos MTO y la categorización ABC para productos MTS, pero otros criterios, como la importancia específica del cliente o pedido, la relación inventario/pronóstico de ventas y el trúput del pedido, se consideran de forma aislada y sin técnica específica.

b) Las órdenes para MTS se lanzan intercaladas con los pedidos para MTO de acuerdo a urgencias, pero ésto hace que el riesgo de retrasos en los pedidos (MTO) y rompimiento de stock en los productos (MTS) sea mayor, pues no se tiene forma de comparar la urgencia de una orden (MTS) con un pedido (MTO).

c) La asignación empírica a cada línea de producción hace que éstas puedan quedar sobrecargadas en ocasiones y desocupadas en otras, disminuyendo la productividad de la planta. Estos problemas pueden ser solucionados con acciones como: a) Calificar las órdenes considerando diferentes criterios y no únicamente la fecha de entrega y la razón inventario/pronóstico de ventas, b) Desarrollar un procedimiento que permita ordenar en forma conjunta las órdenes/pedidos de ambos ambientes (MTS/MTO), y c) Desarrollar un método que permita liberar las órdenes/pedidos (MTS/MTO) de forma que la asignación en cada línea quede balanceada y se pueda maximizar la capacidad.

Para concretar estas acciones, se proponen tres modificaciones al método de programación típico de un ambiente híbrido MTS/MTO, con el fin de evitar los problemas descritos: a) Calificación multi-criterio: Inclusión de más criterios para priorizar las órdenes, b) Regla multi-criterio- GAHPO: Diseñar una regla de secuenciación que incluya los criterios seleccionados para priorizar los productos MTS y los MTO conjuntamente y c) Procedimiento de lotificación de órdenes: Plantear un método de liberación de órdenes que balancee la carga en las diferentes líneas de producción. A continuación se describen las modificaciones propuestas:

a. Calificación multi-criterio. El hecho de que una planta de producción atienda dos mercados con características tan diferentes (MTS y MTO), aumenta la necesidad de considerar diferentes criterios de secuenciación, pues son variadas las medidas de desempeño y la capacidad es compartida por ambos tipos de producto. Las reglas convencionales sólo consideran un aspecto de cada orden, como fecha de entrega, tiempo de proceso o momento de llegada. En un ambiente MTS/MTO donde hay órdenes con exigencias diferentes y las medidas de desempeño son variadas, las reglas simples no logran obtener un resultado óptimo para las diferentes órdenes. Aunque las reglas de secuenciación simples han sido aplicadas ampliamente en la práctica, su eficacia se mantiene limitada debido a su falta de visión global. Las reglas de secuenciación compuestas han demostrado ser más efectivas ya que son construidas por medio de la experiencia humana (Tay y Ho, 2008). Como lo propone (Dabbas, 2001), al combinar múltiples criterios de secuenciación se logra satisfacer simultáneamente múltiples respuestas de interés.

La selección de criterios se hace teniendo en cuenta los indicadores de desempeño que son considerados importantes según el mercado que se atienda. En el mercado MTO es importante 
cumplir con las fechas de entrega prometidas, pero un pedido que genera más trúput que otro debe tener más prioridad, y de igual forma, los clientes más importantes para la empresa tienen primacía sobre otros. En el mercado MTS, aunque mantener el nivel de inventario de productos de categoría $A$ es de vital importancia, cuando un producto de categoría $C$ está cerca de incurrir en faltantes, éste debe tener prioridad, y si una orden va a aportar más trúput que otra, debe ir primero.

b. Regla multi-criterio: Proceso Analítico Jerárquico de Ordenación Grupal (GAHPO). Los métodos de decisión grupal multi-criterio (MAGDM) están atrayendo cada vez más atención en la gestión y han recibido una gran atención de los investigadores (Yue, 2011). Los problemas MAGDM tienen tres características comunes: alternativas, atributos múltiples con unidades inconmensurables y varios expertos, en la que los pesos establecidos por los expertos juegan un papel muy importante. El procedimiento que se llevo a cabo para la aplicación del GAHPO es el siguiente:

i. Jerarquización del modelo: Consiste en descomponer el problema en una jerarquía para facilitar la comprensión.

ii. Selección de expertos: Dado que la calificación de los criterios debe corresponder al beneficio de la empresa como unidad de negocio y no a un área en específico, es importante que todos los involucrados con las decisiones hagan parte de la calificación.

iii. Establecer los pesos de los criterios a partir de juicios de los expertos: Los valores numéricos de calificación(juicios) a utilizar son basados en la tabla sugerida por Saaty, (Camare, 2011). Después de calcular una proporción de consistencia en los juicios de los expertos, utilizar un método de media geométrica para agregación de las calificaciones de estos y calcular un vector de prioridad, se obtiene el peso o ponderación por criterio.

iv. Calificación de cada orden: Con el vector de prioridad calculado, el siguiente paso es dar la calificación a cada orden para la priorización. Para poder hacerlo, cada criterio debe ser normalizado a través de un cambio de magnitud a una escala fija (Pomerol y Barba-Romero, 2000). Con los pesos calculados y normalizados se define una fórmula (regla AHP)en términos de los criterios para calcular la prioridad de cada orden.

c. Procedimiento de lotificación de órdenes para asignación balanceada: El siguiente procedimiento permite lotificar las órdenes de producción con el objetivo de que las diferentes líneas de producción queden con suficiente carga asignada durante todo el período de producción.

a) Cálculo de la carga diaria para cada línea:

Tamaño de Lote Línea ${ }_{i}=\left(\right.$ Unidades programadas Atributo $\left.{ }_{i}\right) /($ Días programados $)$. Para $i=$ tipo de atributo.

b) Definición del lote mínimo por cada orden según sus atributos: Mínimo(Tamaño de Lote Líneai)

c) División de las órdenes en el número de lotes necesarios para no superar el tamaño de lote calculado en paso b) por cada atributo.

d) Ordenar todas las órdenes lotificadas pertenecientes a cada línea de producción de acuerdo a la prioridad calculada con la regla de secuenciación a utilizar, (AHP u otra).

\section{RESULTADOS Y DISCUSION}

Con el fin de comprobar la validez de la metodología propuesta de programación, incluyendo la regla AHP de secuenciación, se desarrolló y experimentó con un modelo de simulación (elaborado mediante el software Promodel) en el cual se representa la planta de producción, con todos los recursos productivos de máquinas y personal que en ella existen, y a través de los cuales se hacen pasar los productos de acuerdo con el orden que determinan las reglas de secuenciación seleccionadas para el estudio, utilizando las siguientes medidas de desempeño del sistema: 
Porcentaje de entrega a tiempo (ET): Se refiere al porcentaje de órdenes despachadas a tiempo. Es la razón entre las órdenes despachadas a tiempo y el total de órdenes despachadas, según lo muestra la expresión (1):

$\mathrm{ET}=\frac{\text { Número de ordenes despachadas a tiempo }}{\text { Ordenesatiempo }+ \text { Ordenes tardías }+ \text { Ordenes anticipadas }} \times 100 \%$

Variabilidad de la entrega $(V E)$ : Se refiere a la variabilidad ponderada promedio del retraso/adelanto de las órdenes entregadas, según la expresión (2):

$V E=\sqrt{\frac{\sum_{i=1}^{n}\left(E R_{i}-F E_{i}\right)^{2}}{n}}$

Donde, $n$ : número total de órdenes, $E R_{i}$ : fecha entrega real de la orden $i$ y FEi: fecha entrega prometida de la orden $i$.

Penalización por tardanza $(P T)$ : La medida consiste en agregarle al retraso los factores de criticidad de importancia y trúput. La penalización se calcula multiplicando el número de días de retraso por un porcentaje del trúput de la orden, porcentaje definido de acuerdo a la importancia del cliente, según la expresión (3):

$\mathrm{PT}=\sum_{\mathrm{i}=1}^{\mathrm{n}} \mathrm{DR}_{\mathrm{i}} \times \mathrm{TR}_{\mathrm{i}} \times \mathrm{IM}_{\mathrm{i}}^{-4}$

Donde, $D R_{i}$ : Días de retraso en la orden $i, T R_{i}$ : trúput de la orden $i, I M_{i}$ : importancia de la orden $i$ Penalización por adelanto $(P A)$ : Es el producto del número de días de adelanto, el número de unidades del pedido y la constante de costo de almacenamiento por unidad, según la expresión (4):

Penalización por adelanto $=\sum_{\mathrm{i}=1}^{\mathrm{n}} \mathrm{DA}_{\mathrm{i}} \times \mathrm{Ud}_{\mathrm{i}} \times \mathrm{C}$

Donde, $D A_{i}$ : días de adelanto en la orden $i, U d_{j}$ : número de unidades de la orden $i, C$ : costo de mantenimiento diario de almacenamiento por unidad.

Penalización por no entrega a tiempo $(P N E)$ : Es la suma de la penalización por retraso y la penalización por adelanto. Esta medida de desempeño es considerada la más importante de todas, ya que como indicador global, considera casi todos los aspectos del desempeño del sistema.

Para esta planta, se identificaron tres criterios generales que se definen de forma diferente para cada mercado (MTS/MTO), según se muestra en la Tabla \# 1. 
Tabla 1: Definición de los criterios de prioridad. Fuente: (Escobar, 2011)

\begin{tabular}{|c|c|c|c|}
\hline \multicolumn{2}{|c|}{ CRITERIO } & MTO & MTS \\
\hline \multirow[t]{2}{*}{ TRÚPUT } & DEFINICIÓN & \multicolumn{2}{|c|}{ Fabricar primero las órdenes que más trúput aporten. } \\
\hline & $\begin{array}{l}\text { UNIDAD DE } \\
\text { MEDIDA }\end{array}$ & \multicolumn{2}{|c|}{ (Precio de venta- Costo variable) x número de unidades a producir. } \\
\hline \multirow[t]{2}{*}{ IMPORTANCIA } & DEFINICIÓN & $\begin{array}{l}\text { Fabricar primero los pedidos de } \\
\text { exportación que por la posibilidad } \\
\text { de generar sobrecostos a la } \\
\text { empresa o roturas de inventario a } \\
\text { los clientes, resulten prioritarios. }\end{array}$ & $\begin{array}{l}\text { Fabricar las órdenes en orden de } \\
\text { su categoría ABC. La clasificación } \\
\text { es definida previamente teniendo } \\
\text { en cuenta factores como el trúput } \\
\text { aportado al año, la importancia } \\
\text { para el canal de distribución, la } \\
\text { participación en el mercado, etc. }\end{array}$ \\
\hline & $\begin{array}{l}\text { UNIDAD DE } \\
\text { MEDIDA }\end{array}$ & $\begin{array}{l}\text { Escala de } 1 \text { a } 10 \text {, siendo } 10 \text { el } \\
\text { más importante. Definida por el } \\
\text { Gerente de Exportaciones al } \\
\text { comienzo del período de } \\
\text { programación. }\end{array}$ & $\begin{array}{l}\text { Escala de } 1 \text { a } 10 \text {, siendo } 10 \text { el más } \\
\text { importante. Definida previamente. }\end{array}$ \\
\hline \multirow[t]{2}{*}{ CUMPLIMIENTO } & DEFINICIÓN & $\begin{array}{l}\text { Fabricar primero los pedidos con } \\
\text { fecha de entrega más próxima. }\end{array}$ & $\begin{array}{l}\text { Fabricar primero las órdenes con } \\
\text { menos días de inventario. }\end{array}$ \\
\hline & $\begin{array}{l}\text { UNIDAD DE } \\
\text { MEDIDA }\end{array}$ & $\begin{array}{l}\text { Días a la fecha de entrega. } \\
\text { Mientras menor el número, mayor } \\
\text { prioridad. }\end{array}$ & $\begin{array}{l}\text { Días de duración del inventario. Se } \\
\text { calcula sacando la relación entre la } \\
\text { existencia actual y el pronóstico de } \\
\text { venta y multiplicando por } 30 \text {. }\end{array}$ \\
\hline
\end{tabular}

Se aplicó el procedimiento GAHPO con los siguientes resultados, (Escobar, 2011):

a. Jerarquización del modelo: La jerarquía se elaboró dando igual peso a la valoración de cada experto, según se muestra en la Figura 1.

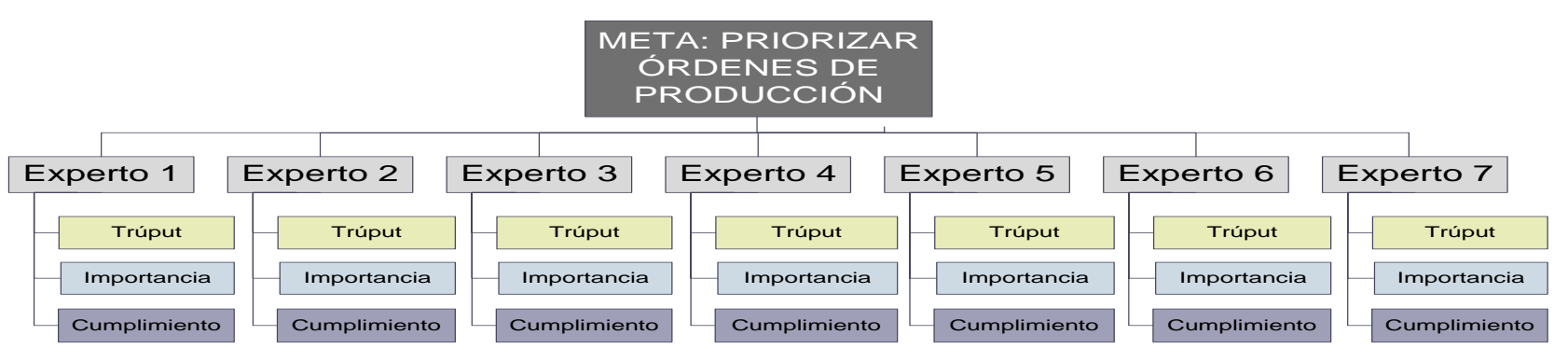

Fig. 1: Jerarquización del problema Multi-Criterio. Fuente (Escobar, 2011)

b. Selección de expertos: El grupo responsable de esta calificación fue compuesto por los siguientes 7 expertos: Presidente General, Gerente de Exportaciones, Gerente de Producto, Gerente de Ventas Nacionales, Gerente Administrativo, Gerente de Producción y Director de Operaciones.

c. Establecer los pesos de los criterios a partir de valoraciones de los expertos: En la Tabla 2 se muestran los pesos asignados a los criterios:

Tabla 2: Pesos definitivos por criterio. Fuente (Escobar, 2011)

\begin{tabular}{|l|r|}
\hline Criterio & Peso \\
\hline Trúput (T) & 0,215 \\
\hline Importancia (I) & 0,335 \\
\hline Cumplimiento $(\mathrm{C})$ & 0,450 \\
\hline Total & 1,000 \\
\hline
\end{tabular}


d. Calificación de cada orden: La fórmula (regla AHP)en términos de los criterios: truput (T), importancia (I) y cumplimiento (C) para calcular la prioridad de cada orden $i$, es dada por la expresión (5):

$\mathbf{P}_{\mathrm{i}}=0,215 . \mathrm{T}_{\mathrm{i}}+0,335 . \mathrm{I}_{\mathrm{i}}+0,45 . \mathrm{C}_{\mathrm{i}}$

Las reglas de secuenciación con las que se comparará la regla AHP, con el fin de evaluar su desempeño, son:

Fecha de entrega más próxima (FEP): se seleccionó esta regla porque la fecha de entrega es el criterio más importante según los requerimientos de los mercados. La fecha de entrega más próxima se define listando los diferentes pedidos con sus fechas de entrega respectivas, y seleccionando primero aquél para el que se haya ofrecido una fecha más cercana a la del momento de la planeación.

Tiempo de procesamiento más corto (TPC): se seleccionó porque es una regla muy recomendada en la literatura. Para su aplicación se listan los pedidos u órdenes de producción con el tiempo de procesamiento calculado para ellos, y se selecciona primero aquél cuyo tiempo sea el menor de la lista.

Mayor trúput (TRUP): se seleccionó porque el trúput es siempre un factor de decisión en la empresa y también aparece en la literatura como regla apropiada para sistemas MTS. El trúput hace referencia al ingreso neto de cada orden o pedido (ingreso - costo variable).

Para la simulación se tomó un histórico de datos de producción correspondiente el año 2011. En total, se simuló la producción de 635 pedidos que se convirtieron en 1988 órdenes de producción y 2690 lotes de producción. Aunque el $57 \%$ de los pedidos corresponden al mercado MTS, el $74 \%$ de la producción total fue destinada al mercado MTO. Las principales motivaciones de simular estos datos, segun tipo y cantidad establecidos, obedecen a que: i) El producto es de consumo estacional: en el mercado MTS, al atender el mercado desde tan cerca, la estacionalidad del consumo se ve reflejada en el inventario de producto terminado, teniendo meses de hasta el doble de ventas que otros. En el mercado MTO, aunque la estacionalidad la absorben en parte las bodegas de los clientes, hay pedidos que se realizan únicamente para cosechas específicas en determinada época del año, ii) Baja frecuencia de los pedidos del exterior: los pedidos de los clientes (MTO), por motivo de reducción de costos de transporte, son generalmente de contenedores completos, lo que hace que algunos clientes pidan pocas veces al año manteniendo el producto en sus bodegas. Las ventas al año por cliente generalmente corresponden a las ventas reales, y iii) El juego de capacidad MTS/MTO cambia en los diferentes meses del año, pues hay unos meses en los que es mucho mayor la producción para el mercado MTS que en otros.

La Tabla 3 muestra el desempeño de cada una de las reglas de secuenciación probada de acuerdo con las medidas de desempeño seleccionadas, incluyendo en la simulación el procedimiento de lotificación. Los resultados muestran un desempeño muy similar de las reglas AHP y FEP, en los criterios entrega a tiempo y variabilidad de la entrega, las cuales resultan superiores a las reglas TCP y TRUP en todos los casos. No obstante, la regla AHP muestra un desempeño significativamente superior en las medias de desempeño asociadas a penalizaciones por tardanza, por adelanto y por no entregar a tiempo.

La Tabla 4 muestra el resumen de los datos de la variable 'Días de Retraso/Adelanto', con el número total de pedidos, pedidos entregados a tiempo (en un rango de una semana de la fecha de entrega), pedidos retrasados y pedidos adelantados, con sus porcentajes respectivos. Las reglas TCP y TRUP tienen porcentajes muy bajos de pedidos a tiempo, aunque TCP tiene un porcentaje muy bajo de pedidos retrasados. Por el contrario las reglas AHP y FEP muestran porcentajes muy similares de alrededor de $63 \%$ de pedidos a tiempo. 
La diferencia entre estas dos reglas se observa en el número de pedidos retrasados y adelantados, en los que la FEP supera a la AHP al tener un menor porcentaje de pedidos retrasados. Estos resultados son muy consecuentes con los criterios de priorización que cada regla considera, pues FEP sólo considera la fecha de entrega para priorización y AHP la considera como el criterio más importante.

Tabla 3: Resumen resultados en cada medida de desempeño. Fuente (Escobar, 2011).

\begin{tabular}{|l|l|l|l|l|}
\hline \multicolumn{1}{|c|}{ Medidas desempeño/Regla } & AHP & FEP & TCP & TRUP \\
\hline$\%$ Entrega a tiempo (ET): & $62.8 \%$ & $63.0 \%$ & $37.5 \%$ & $39.4 \%$ \\
\hline Variabilidad en la entrega (VE): & 7.66 & 7.76 & 12.12 & 12.04 \\
\hline Penalización por tardanza (PT): & $\$ 10,625,628$ & $\$ 23,934,067$ & $\$ 62,949,939$ & $\$ 42,477,673$ \\
\hline Penalización por Adelanto (PA): & $\$ 23,095,800$ & $\$ 19,189,000$ & $\$ 60,348,200$ & $\$ 56,543,000$ \\
\hline $\begin{array}{l}\text { Penalización por no entrega a } \\
\text { tiempo (PNE): }\end{array}$ & $\$ 33,721,428$ & $\$ 43,123,067$ & $\$ 123,298,139$ & $\$ 99,020,673$ \\
\hline
\end{tabular}

Tabla 4: Resumen de pedidos a tiempo, retrasados y adelantados. Fuente (Escobar, 2011)

\begin{tabular}{|l|r|r|r|r|r|r|r|r|}
\hline \multicolumn{1}{|c|}{ Pedidos } & \multicolumn{7}{c|}{ \# de pedidos \% de pedidos } \\
\hline Pedidos/Regla & \multicolumn{2}{|c|}{ AHP } & \multicolumn{2}{|c|}{ FEP } & \multicolumn{2}{|c|}{ TCP } & \multicolumn{2}{|c|}{ TRUP } \\
\hline Pedidos totales & 635 & $100 \%$ & 635 & $100 \%$ & 635 & $100 \%$ & 635 & $100 \%$ \\
\hline Pedidos a tiempo & 399 & $62.8 \%$ & 400 & $63.0 \%$ & 238 & $37.5 \%$ & 250 & $39.4 \%$ \\
\hline Pedidos retrasados & 67 & $10.6 \%$ & 55 & $8.7 \%$ & 55 & $8.7 \%$ & 215 & $33.9 \%$ \\
\hline $\begin{array}{l}\text { Pedidos } \\
\text { adelantados }\end{array}$ & 169 & $26.6 \%$ & 180 & $28.3 \%$ & 342 & $53.9 \%$ & 170 & $26.8 \%$ \\
\hline
\end{tabular}

La Tabla 5 muestra el número de días de retraso y el número de días de adelanto, junto con la variabilidad de los días de retraso/adelanto. Los datos muestran como, tanto los días de retraso como los de adelanto de la regla AHP son menores que los de la regla FEP, lo que se ve reflejado en la variabilidad del retraso/adelanto. Esto indica que los resultados de la regla AHP se encuentran más cercanos al cero (pedido a tiempo), objetivo máximo del ejercicio.

Tabla 5: Resumen de días totales de retraso y adelanto. Fuente (Escobar, 2011)

\begin{tabular}{|l|c|c|c|c|}
\hline & AHP & FEP & TCP & TRUP \\
\hline Días totales de retraso & 101 & 189 & 41 & 1234 \\
\hline Días totales de adelanto & 540 & 601 & 3094 & 616 \\
\hline $\begin{array}{l}\text { Variación } \\
\text { retraso/adelanto }\end{array}$ & 7.66 & 7.76 & 12.12 & 12.04 \\
\hline
\end{tabular}

La Tabla 6 muestra los porcentajes de pedidos a tiempo, retrasados y adelantados por MTS y MTO. Se observa que las reglas AHP y FEP tienen un comportamiento similar en ambos mercados, mostrando levemente mejores resultados en el mercado MTO, siendo más fuerte la diferencia en la regla FEP. La regla TRUP es la que mayor diferencia presenta, reportando mayores retrasos en las órdenes MTS que en las MTO. Esto es explicable al considerar que las órdenes MTS generalmente son más pequeñas y aportan menos trúput, por lo que la regla hace que éstas se procesen de últimas y presenten más retrasos. En la regla TCP ocurre lo contrario, pues como las órdenes MTS son de procesamiento más rápido debido a su tamaño, éstas se procesan primero y presentan menos retrasos. 
Tabla 6: Porcentaje de pedidos a tiempo según MTS/MTO. Fuente (Escobar, 2011)

\begin{tabular}{|l|l|l|l|l|}
\hline & \multicolumn{4}{|c|}{ MTS / MTO } \\
\hline$\%$ Pedidos/Regla & \multicolumn{1}{|c|}{ AHP } & \multicolumn{1}{|c|}{ FEP } & \multicolumn{1}{|c|}{ TCP } & \multicolumn{1}{|c|}{ TRUP } \\
\hline$\%$ pedidos a tiempo & $60.7 \% / 65.7 \%$ & $58.2 \% / 69.4 \%$ & $37.1 \% / 38.0 \%$ & $39.0 \% / 39.9 \%$ \\
\hline$\%$ pedidos retrasados & $14.0 \% / 5.9 \%$ & $12.1 \% / 4.1 \%$ & $6.9 \% / 11.1 \%$ & $42.3 \% / 22.5 \%$ \\
\hline$\%$ pedidos adelantados & $25.3 \% / 28.4 \%$ & $29.7 \% / 6.6 \%$ & $56.0 \% / 50.9 \%$ & $18.7 \% / 7.6 \%$ \\
\hline
\end{tabular}

Con todo, los datos de entregas a tiempo por sí solos no son suficientes pues no muestran la criticidad del retraso en función de la importancia del cliente o del pedido o de la contribución del trúput.

Al analizar la penalización separando los datos MTS de los MTO (Tabla 7), se observa que la penalización total es aproximadamente la misma, pero desagregada presenta diferencias considerables. Tanto en la orden AHP como en la FEP, la penalización por adelanto de las órdenes MTO es la que mayor diferencia presenta.

Tabla 7: Penalización en MTS y MTO en pesos. Fuente (Escobar, 2011)

\begin{tabular}{|c|l|r|r|r|r|}
\hline \multirow{2}{*}{ Penalización Total } & \multicolumn{1}{c|}{ AHP } & \multicolumn{1}{c|}{ FEP } & \multicolumn{1}{c|}{ TCP } & \multicolumn{1}{c|}{ TRUP } \\
\cline { 2 - 6 } & MTS & $\$ 3,721,428$ & $\$ 43,123,067$ & $\$ 123,298,139$ & $\$ 99,020,673$ \\
\hline \multirow{2}{*}{ MTS } & Total & $\$ 16,983,605$ & $\$ 26,002,079$ & $\$ 67,804,261$ & $\$ 40,575,400$ \\
\cline { 2 - 6 } & Retraso & $\$ 9,177,805$ & $\$ 20,912,079$ & $\$ 52,054,461$ & $\$ 31,669,600$ \\
\cline { 2 - 6 } & Adelanto & $\$ 7,805,800$ & $\$ 5,090,000$ & $\$ 15,749,800$ & $\$ 8,905,800$ \\
\hline \multirow{2}{*}{ MTO } & Total & $\$ 16,737,823$ & $\$ 17,120,988$ & $\$ 55,493,878$ & $\$ 58,445,273$ \\
\cline { 2 - 6 } & Retraso & $\$ 1,447,823$ & $\$ 3,021,988$ & $\$ 10,895,478$ & $\$ 10,808,073$ \\
\cline { 2 - 6 } & Adelanto & $\$ 15,290,000$ & $\$ 14,099,000$ & $\$ 44,598,400$ & $\$ 47,637,200$ \\
\hline
\end{tabular}

A pesar de que la penalización total es similar, entre AHP y FEP, la diferencia principal se encuentra en la penalización por retrasos, la cual representa el $32 \%$ de la penalización total en AHP y el $56 \%$ en FEP. Esto quiere decir que los pedidos con retraso en la regla FEP $(8.7 \%$ del total) pertenecían a órdenes de mayor importancia o mayor trúput que los pedidos que presentaron retraso al utilizar la regla AHP (10.6\% del total). Esta idea se puede ver más claramente en la Figura 2.

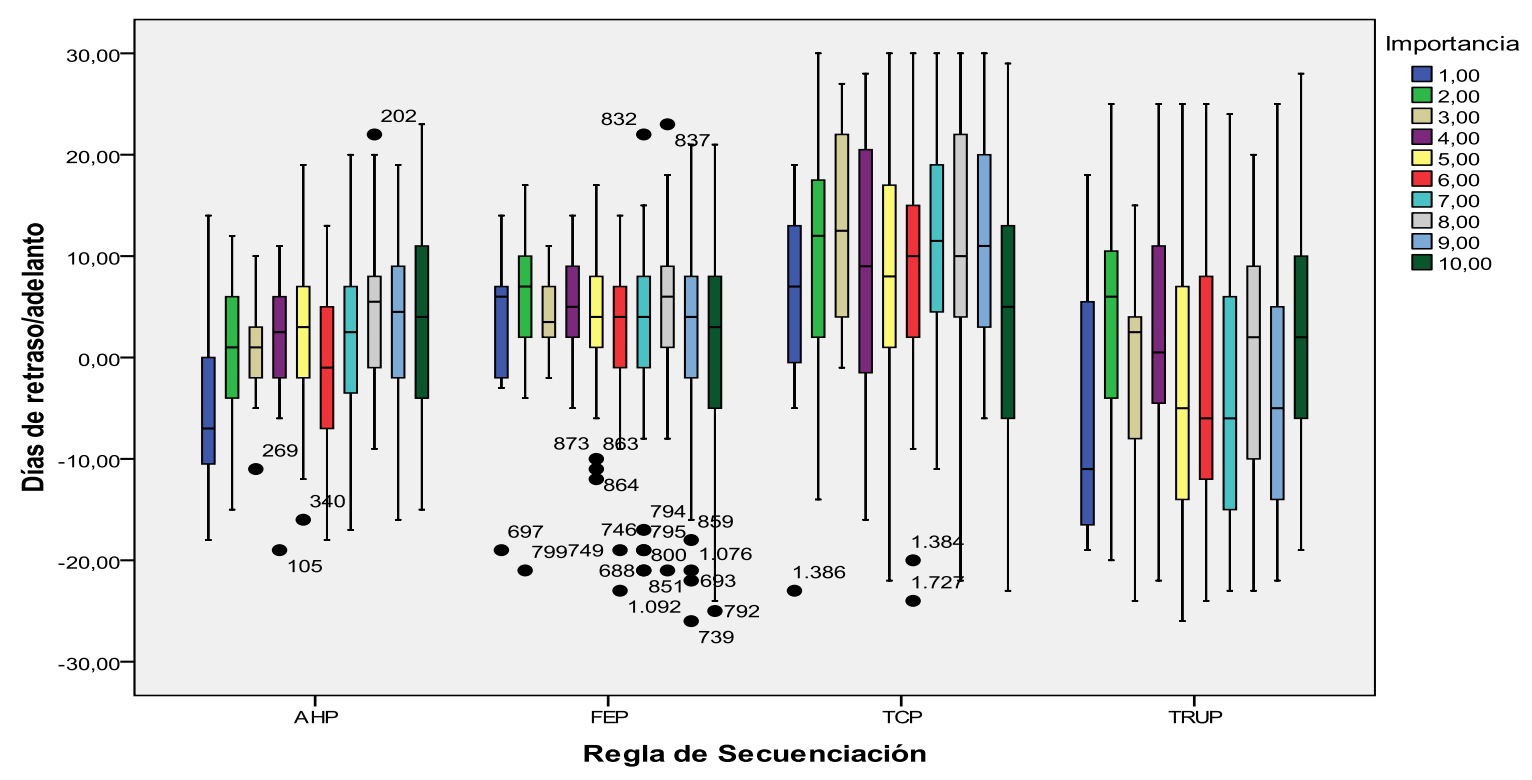

Fig.2: Diagrama de cajas de días de retraso por regla, agrupado por importancia del pedido.Fuente (Escobar, 2011) 
El Diagrama de Cajas muestra los días de retraso/adelanto, agrupando por regla de secuenciación y categorizando en las cajas la importancia de la orden. La gráfica mencionada muestra que la regla AHP presenta resultados que van mejorando a medida que aumenta la importancia. La regla FEP muestra múltiples datos atípicos, la mayoría como días de retraso y en órdenes de importancia mayor a 5 . Esas órdenes pueden ser muy críticas, pues si son clientes de categoría 10 , estos retrasos podrían debilitar la relación con un cliente tan importante.

\section{CONCLUSIONES}

La regla de prioridad propuesta en el presente trabajo se constituye en el principal resultado, pues además de realizar una contribución a lo ya existente en la literatura, considera características específicas de las empresas del entorno local y medidas de desempeño aceptadas en el entorno para su construcción y validación. Con la validación realizada puede concluirse que el objetivo propuesto se alcanza en forma sobresaliente, pues la regla de prioridad desarrollada demuestra tener un resultado superior en las medidas de desempeño seleccionadas. Esto significa para la empresa la posibilidad de una metodología de programación y control de su producción más eficiente desde el punto de vista de la utilización de la capacidad, los costos y los niveles de satisfacción de sus clientes.

La validación de la regla de secuenciación propuesta permite demostrar la superioridad de su desempeño a partir de la medición de variables respuesta de validez universal como el tiempo de retraso y el trúput, además de una medida de desempeño construida para este propósito específico. Este resultado permite concluir que siempre es posible obtener desarrollos propios, adaptados a cada ambiente específico, que permitan acercar las empresas regionales a objetivos de desempeño competitivo superiores.

Aunque la regla FEP presenta menores retrasos, la regla AHP mostró mejores resultados generales ya que considera la importancia y el trúput para la priorización. La regla FEP presenta altos riesgos de obtener malos resultados en órdenes de la máxima importancia, lo cual es algo crítico para los objetivos definidos. La regla AHP, al considerar esta importancia como un criterio de priorización, disminuye este riesgo. 69

Las reglas TCP y TRUP tienen objetivos "internos" y no con énfasis en el mercado, pues no consideran la fecha de entrega ni la importancia de los clientes o pedidos, por lo que la penalización obtenida es muy alta. La validación de la regla de secuenciación propuesta permite demostrar la superioridad de su desempeño a partir de la medición de variables respuesta de validez universal como el tiempo de retraso y el trúput, además de una medida de desempeño construida para este propósito específico.

Este resultado permite concluir que es posible obtener desarrollos propios, adaptados a cada ambiente específico, que permitan acercar las empresas regionales a objetivos de desempeño competitivo superiores. Los criterios utilizados para la priorización son los específicos para los requerimientos del caso de estudio, pero si estos se modifican para la realidad de otra empresa, el método es aplicable en otros casos con características similares.

\section{REFERENCIAS}

Camare, $\mathrm{H} .:$ Multicriteria decision evaluation of adaptation strategies for vulnerable coastal communities,p.1-65.(April 2010), Retrieved from http://www.ruor.uottawa.ca/fr/handle/10393/20112) (2011).

Castrillón, O., Sarache, W. y Giraldo, J: Aplicación de un Algoritmo Evolutivo en la Solución de Problemas Job Shop-Open Shop. Información Tecnológica., vol.22, no.1, p.83-92. (2011).

Cattani, K., Dahan, E., y Schmidt, G., Texturing: Using Make-to-Order Production Layered on Make-to-Stock Production to Hedge Against Uncertainty. (2003). Under Review at Journal of Operations Management. 
Chang, S., Heuristic PAC model for hybrid MTO and MTS production environment. International Journal of Production Economics, Vol. 85, No. 3, p. 347-358. (2003).

Chen, C., Mestry, S., Damodaran, P., y Wang, C., The capacity planning problem in make-to-order enterprises. Mathematical and Computer Modelling, Vol. 50, No. 9-10, p. 1461-1473. (2009).

Corti, D., Pozzetti, A., y Zorzini, M., A capacity-driven approach to establish reliable due dates in a MTO environment. International Journal of Production Economics, Vol. 104, No. 2, p. 536-554. (2006).

Dabbas, R., A combined dispatching criteria approach to scheduling semiconductor manufacturing systems. Computers and Industrial Engineering, Vol. 39, No. 3-4, p. 307-324. (2001).

Dong, Y., Zhang, G., Hong, W., y Xu, Y., Consensus models for AHP group decision making under row geometric mean prioritization method. Decision Support Systems, Vol. 49, No. 3, p. 281-289. (2010).

Escobar, M., A note on AHP group consistency for the row geometric mean priorization procedure. European Journal of Operational Research, Vol. 153, No.2, p.318-322. (2004).

Escobar, P., Rediseño del proceso de programación detallada en un ambiente make-tostock/make-to-order: Caso de una empresa metalmecánica de Manizales (Colombia). Tesis de Maestría en Ingeniería Industrial, Universidad Nacional de Colombia.(2011)

Federgruen, A., y Katalan, Z., The impact of adding a make-to-order item to a make-to-stock production system. Management Science, Vol. 45, No. 7, p. 980-994.(1999).

Gharehgozli, A. y Rabbani, M., A Proposed Hierarchical Production Planning Structure for Combined MTS / MTO Environments Based on Order Penetration Point. Proceedings of the 9th WSEAS Int. Conf. on Applied Mathematics, Istanbul, Turkey, May 27-29, pp. 146-151, (2006).

Holthaus, Oliver, y Rajendran, C., New dispatching rules for scheduling in a job shop_An experimental study. The International Journal of Advanced Manufacturing Technology, Vol. 13, No. 2, p.148-153. (1997).

Ishizaka, A., y Labib, A., Selection of new production facilities with the Group Analytic Hierarchy Process Ordering method. Expert Systems with Applications, Vol. 38, No.11, p.14336-14345. (2011a).

Ishizaka, A., y Labib, A., Review of the main developments in the analytic hierarchy process. Expert Systems with Applications, Vol. 38, No. 11, p. 14336-14345. (2011b).

Jiang, L., y Geunes, J., Impact of introducing make-to-order options in a make-to-stock environment. European Journal of Operational Research, Vol. 174, No. 2, p. 724-743. (2006).

Kalantari, M., Rabbani, M., y Ebadian, M.: A decision support system for order acceptance/rejection in hybrid MTS/MTO production systems. Applied Mathematical Modelling, Vol. 35, No. 3, p. 1363-1377. (2011).

Kaminsky, P. y Kaya, O., MTO-MTS Production Systems in Supply Chains. Proceedings of 2006 NSF Design, Service, and Manufacturing Grantees and Research Conference, St. Louis, Missouri. Retrieved from: http://citeseerx.ist.psu.edu/ (2011).

Kerkkanen, A., Determining semi-finished products to be stocked when changing the MTS-MTO policy: Case of a steel mill. International Journal of Production Economics, Vol. 108, No. 1-2, p. 111-118. (2007). 
Lin, J., Wang, F., y Chang, Y., A hybrid push/pull-dispatching rule for a photobay in a 300mm wafer fab. Robotics and Computer-Integrated Manufacturing, Vol. 22, No. 1, p. 47-55. (2006).

Meredith, J., y Akinc, U., Characterizing and structuring a new make-to-forecast production strategy. Journal of Operations Management, Vol.25, No. 3, p. 623-642. (2007).

Miltenburg, J., Manufacturing Strategy: How to formulate and Implement a Winning Plan 2nd ed. Productivity Press. New York. (2005).

Olhager, J., Long-term capacity management: Linking the perspectives from manufacturing strategy and sales and operations planning. International Journal of Production Economics, Vol. 69 , No. 2, p. 215-225. (2001).

Pomerol, J. y Barba-Romero, S., Multicriterion decision in management: principles and practice. Springer, Ed. Norwell, Massachusetts (2000).

Rafiei, H., y Rabbani, M., Order Partitioning in Hybrid MTS / MTO Contexts using Fuzzy ANP. World Academy of Science, Engineering and Technology, Vol. 58, p. 467-472. (2009a).

Rafiei, H., y Rabbani, M., An MADM Framework toward Hierarchical Production Planning in Hybrid MTS / MTO Environments. World Academy of Science, Engineering and Technology, Vol. 58, p. 462-467.(2009b).

Rajagopalan, S., Make to Order or Make to Stock: Model and Application. Management Science, Vol. 48, No. 2, p. 241-256. (2002).

Rajendran, C., y Holthaus, O., A comparative study of dispatching rules in dynamic flowshops and jobshops. European Journal of Operational Research, Vol.116, No. 1, p. 156-170. (1999).

Soman, C., Combined make-to-order and make-to-stock in a food production system. International Journal of Production Economics, Vol.90, No. 2, p. 223-235. (2004).

Soman, C., Pietervandonk, D., y Gaalman, G., Comparison of dynamic scheduling policies for hybrid make-to-order and make-to-stock production systems with stochastic demand. International Journal of Production Economics, Vol.104, No. 2, p. 441-453. (2006).

Soman, C., Vandonk, D., y Gaalman, G., Capacitated planning and scheduling for combined make-to-order and make-to-stock production in the food industry: An illustrative case study. International Journal of Production Economics, Vol. 108, No. 1-2, p. 191-199. (2007).

Tay, J., y Ho, N., Evolving dispatching rules using genetic programming for solving multi-objective flexible job-shop problems. Computers \& Industrial Engineering, Vol. 54, No. 3, p. 453-473. (2008).

Tsubone, H., Ishikawa, Y., y Yamamoto, H., Production planning system for a combination of make-to-stock and make-to-order products. International Journal of Production Research, Vol.40, No. 18 , p. $4835-4851$ (2002).

Wu, M., Jiang, J., y Chang, W., Scheduling a hybrid MTO/MTS semiconductor fab with machinededication features. International Journal of Production Economics, Vol.112, No. 1, p. 416-426. (2008).

Zaerpour, N., Rabbani, M., Gharehgozli, A., y Tavakkoli-Moghaddam, R., Make-to-order or maketo-stock decision by a novel hybrid approach. Advanced Engineering Informatics, Vol. 22, No. 2, p. 186-201. (2008). 